$\mathrm{E}$

EVALUAR
2021, Vol. 21, No. 2

ISSN $1667-4545$

Recuperado de https://revistas.unc.edu.ar/index.php/revaluar

Laboratorio de Evaluación Psicológica y Educativa

Facultad de Psicología - Universidad Nacional de Córdoba

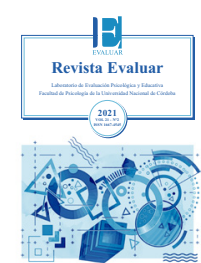

\title{
Validación de Child and Adolescent Mindfulness Measure en escolares de Lima, Perú
}

\section{Validation of Child and Adolescent Mindfulness Measure in pupils from Lima, Peru}

\author{
María Graciela Gustin-García ${ }^{1}$, Alberto Alegre-Bravo * 2 \\ 1 - Universidad Antonio Ruiz de Montoya, Pueblo Libre, Perú. \\ 2 - Universidad San Ignacio de Loyola, Lima, Perú.
}

Recibido: 23/10/2020 Revisado: 15/12/2020 Aceptado: 17/02/2021
Introducción

Método

Resultados

Discusión

Referencias

\section{Resumen}

El presente estudio tuvo como objetivo adaptar y analizar las propiedades psicométricas de la escala Child and Adolescent Mindfulness Measure - CAMM de (Greco, Baer, \& Smith, 2011) en una muestra de 2120 estudiantes de ambos sexos, entre 10 y 17 años, ubicados en colegios públicos y privados de Lima Metropolitana. Se realizaron los análisis factoriales, exploratorio y confirmatorio a la escala adaptada lingüísticamente y se evidenció una estructura unifactorial para 8 ítems de los 10 de la escala original, con una varianza explicada del $74 \%$. Con el análisis de confiabilidad se obtuvo como resultado una adecuada consistencia interna, siendo el alfa ordinal de .78. Adicionalmente, se analizaron diferencias entre los puntajes obtenidos según sexo y edad. Se concluyó que la versión con 8 ítems de la escala CAMM adaptada posee evidencias de validez y de confiabilidad para evaluar mindfulness/atención plena en niños y adolescentes limeños.

Palabras clave: mindfulness, adaptación lingüística, confiabilidad, validez

\begin{abstract}
The aim of this study was to translate and study the psychometric properties of the Child and Adolescent Mindfulness Measure - CAMM (Greco, Baer, \& Smith, 2011). The CAMM was administered to a sample of 2120 students in Lima Metropolitana, boys and girls aged between 10 and 17 years old. After an exploratory and confirmatory factor analysis a single component structure showed good fit with an explained variance of $74 \%$. The CAMM showed an adequate internal consistency $\left(\alpha_{\text {ord }}=.78\right)$. Moreover, differences between the scores obtained by gender and age were analyzed. The new Spanish version of the CAMM with 8 items showed adequate psychometric properties, so it can be used with children and teenagers from Lima Metropolitana.
\end{abstract}

Keywords: mindfulness, linguistic adaptation, consistency, validity

\footnotetext{
* Correspondencia a: Alberto Alegre-Bravo. E-mail: aalegrebavo@gmail.com

Cómo citar este artículo: Gustin-García, M. G., Alegre-Bravo, A. (2021). Validación de Child and Adolescent Mindfulness Measure. Revista Evaluar, 21(2), 63-79. Recuperado de https://revistas.unc.edu.ar/index.php/revaluar

Participaron en la edición de este artículo: Julian Narvaja, Stefano Macri, Juan Cruz Balverdi, Alicia Molinari, Florencia Ruiz, Ricardo Hernández.
} 


\section{Introducción}

Mindfulness o atención plena (Simón, 2010) ha despertado el interés en las últimas décadas debido a los múltiples beneficios a los cuales ha sido asociada. Su rápida difusión se ha extendido a diversos ámbitos como el médico, educativo, neurocientífico y psicológico, entre otros. A su vez, la literatura científica que avala la eficacia del uso de técnicas basadas en atención plena se encuentra progresivamente en aumento (Baer, Carmody, \& Hunsinger, 2012; Cunha, Galhardo, \& Pinto-Gouveia, 2013; Brown, Ryan, \& Creswell, 2007). A medida que las investigaciones acerca de la eficacia de los programas basados en atención plena dirigidos a niños y a adolescentes en contextos clínicos y educativos se incrementan, la necesidad de contar con una medida válida y confiable para la evaluación de mindfulness disposicional es crucial (Baer et al., 2012; Bergomi, Tschacher, \& Kupper, 2013; Greco, Baer, \& Smith, 2011; de Bruin et al., 2013).

Se han construido y validado una amplia variedad de herramientas de medición, principalmente dirigidas a adultos; la mayoría de ellas han sido diseñadas para evaluar la tendencia disposicional o mindfulness rasgo. Desde el campo de la psicología se han desarrollado varios métodos para evaluar y estudiar diversas variables de la conducta humana que no son susceptibles de ser observadas directamente, sino que lo son primariamente por la persona que los experimenta, como es el caso de mindfulness rasgo o disposicional (Baer, 2011). En este sentido, el uso de los cuestionarios de tipo autoinforme puede proporcionar información válida y confiable; ya que uno de los objetivos centrales en el uso de estos en el área de investigación es evaluar los cambios que ocurren después de una intervención basada en mindfulness o de una práctica más a largo plazo (Baer, 2011; Brown \& Ryan, 2003).
Los instrumentos existentes para adultos responden tanto a modelos unifactoriales como multifactoriales en la conceptualización de atención plena (Baer, 2011; Bergomi et al., 2013; Brown \& Ryan, 2003).

El enfocar todos los esfuerzos en crear herramientas para adultos ha dado lugar a un vacío o déficit en la existencia de herramientas para niños (Bergomi et al., 2013; de Bruin et al., 2013; Greco et al., 2011; Sauer et al., 2012). Actualmente, son pocos los instrumentos existentes para medir mindfulness en esta población (Baer et al., 2006; Brown, West, Loverich, \& Biegel, 2011; Greco et al., 2011; Johnson, Burke, Brinkman, \& Wade, 2017; Lawlor et al., 2014; León del Barco, Martín-López, García-Martín, \& Felipe, 2008) y específicamente en Perú no se encuentran aún instrumentos que midan dicha variable y que cuenten con estudios previos de validez y confiabilidad.

Ante esta necesidad se ha identificado a la escala Child and Adolescent Mindfulness Measure - CAMM de Greco et al. (2011) como una medida del constructo disponible para niños $\mathrm{y}$ adolescentes. A continuación, se detalla su proceso de construcción y sus validaciones a nivel internacional.

Para la construcción y validación inicial del CAMM, realizada en Estados Unidos, los autores de dicho instrumento realizaron cuatro estudios con el objetivo de demostrar su validez y confiabilidad.

En el primer estudio $(\mathrm{N}=428)$ se creó un pool de reactivos basándose en la conceptualización que Baer et al. (2004) postularon sobre el mindfulness cuando desarrollaron para la población adulta el Inventario Kentucky de Habilidades de Mindfulness (KIMS; por sus siglas en inglés Kentucky Inventory of Mindfulness Skills). Baer et al. (2004) lo definieron como una habilidad compuesta por cuatro facetas diferentes, pero relacionadas entre sí, denominadas: observación 
(el grado de atención o de darse cuenta de los fenómenos internos como sensaciones corporales, pensamientos y sentimientos), descripción (la capacidad de detallar verbalmente las experiencias internas), actuar con atención (la capacidad de darse cuenta y de tener un compromiso con la actividad que se está realizando) y aceptación (la capacidad para tomar una actitud de apertura para experimentar eventos externos e internos sin emitir juicios acerca de ellos). Sin embargo, los autores del CAMM no incluyeron la faceta de descripción porque no se consideró apropiada para el nivel evolutivo y de desarrollo propio de niños y adolescentes. Es decir, al encontrarse esta población específica en un proceso de maduración y desarrollo de sus habilidades cognitivas y lingüísticas, no resultó pertinente tomar en cuenta dicha faceta (Greco et al., 2011).

Esta primera versión de la escala CAMM compuesta por un grupo de 25 ítems en un formato de autoinforme y con una escala Likert de cinco puntos $(0=$ Nunca es verdadero a $4=$ Siempre es verdadero) fue revisada por expertos en el área de la psicología clínica y de programas de intervención basados en atención plena para niños y adolescentes. Se aplicó esta primera versión a una muestra de 428 niños y adolescentes entre $10 \mathrm{y}$ 17 años. Los enunciados que no fueron comprendidos por más del $2 \%$ de los participantes fueron modificados (Greco et al., 2011).

En el segundo estudio $(\mathrm{N}=334)$, se analizó la estructura factorial de la escala con los 25 reactivos construidos durante la fase previamente descrita. Se realizó un análisis de componentes principales con una rotación oblicua para permitir intercorrelaciones entre los factores, examinándose soluciones tanto bifactoriales como trifactoriales, tal y como fue propuesto por la conceptualización de mindfulness realizada por Baer et al. (2004). Se halló que una solución unifactorial compuesta por 10 ítems resultaba acertada y pertinente, ade- más, el análisis de consistencia interna arrojó un coeficiente alfa de Cronbach de .80 (Greco et al., 2011).

En el tercer estudio $(\mathrm{N}=332)$, la solución unifactorial identificada en los análisis previos fue puesta a prueba utilizando un análisis factorial confirmatorio y se encontró evidencia que respaldó dicho resultado, ya que los índices de adecuación mostraron un buen ajuste y que la solución factorial de un solo factor era la más apropiada $(\mathrm{RMSEA}=.07, \mathrm{SRMR}=.06, \mathrm{CFI} .=90 \mathrm{y}$ NNFI $=.80$; Greco et al., 2011).

En el cuarto estudio $(\mathrm{N}=319)$, se realizó un análisis de la validez convergente y divergente de la prueba y para ello se utilizaron los siguientes instrumentos: Children's Somatization Inventory-Short Form (CSI-SF; Walker \& Garber, 2001), Symptoms and Functioning Scale (SFS; Bickman, 2006) Youth Quality of Life-Revised (YQOL-R; Patrick, Edwards, \& Topolski, 2002), Avoidance and Fusion Questionnaire for Youth (AFQ-Y; Greco, Lambert, \& Baer, 2008), White Bear Suppression Inventory (WBSI; Wegner \& Zanakism, 1994), Social Skills Rating System-Teacher Form (SSRS-TF; Gresham \& Elliot, 1990). Greco et al. (2011) encontraron que los puntajes de la escala CAMM correlacionan significativa y positivamente con resultados favorables como calidad de vida, habilidades sociales y con un óptimo rendimiento académico; asimismo correlacionan negativamente con quejas somáticas y con procesos no adaptativos, tales como falta de flexibilidad psicológica, supresión de pensamientos y síntomas internalizados (ansiedad, timidez, retraimiento y depresión) y externalizados (trastornos conductuales).

Finalmente, los autores del CAMM al analizar sus muestras no encontraron diferencias significativas de acuerdo con las variables sociodemográficas tales como sexo, edad, raza y grado de escolaridad (Greco et al., 2011). 
La evidencia mostrada por Greco et al. (2011) acerca de la escala CAMM como un instrumento de medición coherente y pertinente para niños y adolescentes generó que diversos investigadores se interesaron en ella. Se puede observar que desde la publicación del CAMM por Greco et al. (2011) se han registrado varios estudios psicométricos de este instrumento. Todas las adaptaciones de la escala original en inglés a otros idiomas realizadas previamente utilizaron el método de retro-traducción para asegurar la equivalencia semántica y conceptual del instrumento; a continuación, se describirán algunas de ellas (Chiesi et al., 2016; Cunha et al., 2013; de Bruin et al., 2013; Dion et al., 2017; Saggino et al., 2017; Viñas et al., 2015).

En Portugal (Cunha et al., 2013) se halló una estructura unifactorial y se eliminó uno de los ítems originales de la prueba, mientras que en Holanda (de Bruin et al., 2013) también se halló una estructura unifactorial $(\alpha=.80)$ y se mantuvieron todos los ítems. Posteriormente, en España, una versión en catalán (Viñas, Malo, González, Navaro, \& Casas, 2015), también en Australia (Kuby et al., 2015), en Canadá, una versión francesa (Dion, Paquette, Daigneault, Godbout, \& Hébert, 2017) y dos adaptaciones en Italia (Chiesi et al., 2016; Saggino et al., 2017) evidenciaron la misma estructura unidimensional a pesar de que, en algunos casos, ciertos ítems fueron eliminados.

Ya sea el CAMM en su versión de 10 ítems (Greco et al., 2011; de Bruin et al., 2013; Dion et al., 2017; Kuby et al., 2015; Viñas et al., 2015), de 9 (Cunha et al., 2013) o de 8 ítems (Chiesi et al., 2016; Saggino et al., 2017), todos ellos han evidenciado igualmente la estructura unifactorial de esta escala.

Las muestras utilizadas en diversos estudios variaron ligeramente en cuanto a los rangos de edad de los participantes. La escala CAMM ha sido aplicada a estudiantes entre los 9 y 19 años:
12 a 18 años (Cunha et al., 2013), 10 a 16 años (de Bruin et al., 2013) 11 a 16 años (Viñas et al., 2015) 12 a 15 años (Kuby et al., 2015). Dion et al. (2017) trabajaron con adolescentes entre los 15 y 19 años, mientras que Saggino et al. (2017) incluyeron sujetos con un rango de edad entre 9 y 18 años.

Los estudios que se realizaron evidenciaron una adecuada consistencia interna y todos ellos han obtenido buenos índices de coeficiente alfa de Cronbach. Asimismo, algunos de estos estudios psicométricos informan valores adecuados en la correlación test-retest, revelando una adecuada estabilidad en los puntajes, con un mes de intervalo (Cunha et al., 2013; Dion et al., 2017), con dos semanas (Dion et al., 2017) y con un año de intervalo (Viñas et al., 2015).

Los estudios psicométricos revisados sugieren que la escala de medición CAMM se ajusta a un modelo unidimensional con buenas propiedades psicométricas que puede ser utilizada para evaluar mindfulness rasgo en contextos clínicos, educativos y de investigación con niños y adolescentes entre los 9 y 18 años.

Debido a que se carece de un instrumento adaptado y validado para la población peruana, esta investigación tuvo como propósito principal realizar una adaptación lingüística y cultural del instrumento Child and Adolescent Mindfulness Measure - CAMM (Greco et al., 2011) y estudiar sus propiedades psicométricas con la finalidad de que pueda ser una herramienta útil para evaluar mindfulness disposicional, también denominado mindfulness rasgo, en niños y adolescentes entre los 10 y 17 años. Asimismo, este estudio se considera un aporte a la investigación de las propiedades psicométricas de la escala CAMM en ámbitos socioculturales diferentes al de la creación de su versión original (Greco et al., 2011; Cunha et al., 2013 y de Bruin et al., 2013). 


\section{Método}

El presente constituye un estudio instrumental (Montero \& León, 2002), pues se orienta a adaptar lingüísticamente la escala Child and Adolescent Mindfulness Measure - CAMM y determinar sus propiedades psicométricas en niños y adolescentes de Lima.

\section{Participantes}

Se contó con la participación de 2120 estudiantes de ambos sexos (hombres $=680$ y mujeres =1440) pertenecientes a la educación básica regular, desde quinto grado de primaria hasta quinto de secundaria, de colegios de Lima Metropolitana (públicos $=825$ y privados $=1295$ ). Las edades de los participantes se encontraban entre los $10 \mathrm{y}$ 17 años $(\mathrm{M}=13.50, \mathrm{DE}=1.92)$ dado que la prueba fue construida para esas edades específicas.

\section{Instrumento}

El instrumento Child and Adolescent Mindfulness Measure (CAMM; Greco et al., 2011) es un cuestionario de autoinforme que consta de 10 ítems inversos de simple y rápida administración y que puede ser aplicado en forma individual o grupal y está dirigido a niños a partir de 10 años. El formato de respuesta se presenta en una escala Likert de 5 puntos con un rango entre $0=$ Nunca es verdadero y $4=$ Siempre es verdadero. El sujeto debe puntuar el grado en que se siente identificado con cada reactivo. Las alternativas de respuesta son: 0 (Nunca es verdadero), 1 (Rara vez es verdadero), 2 (A veces es verdadero), 3 (Frecuentemente es verdadero), 4 (Siempre es verdadero).

Para obtener la puntuación total de la escala, se asignan puntajes inversos a cada respuesta de la siguiente manera: Al 0 le corresponden 4 puntos, al 1 le corresponden 3, al 2 la puntuación 2, al 3 se le asigna puntaje 1 y al 4 le corresponde el 0 . Los resultados se calculan mediante la suma de la puntuación de cada ítem invertido dando lugar a un puntaje total de entre 0 y 40 puntos. Dado que se trata de una escala de tipo Likert, a mayor valor obtenido, mayor nivel en el rasgo medido (mindfulness).

La escala CAMM se tradujo al español siguiendo el método de traducción y retro-traducción o traducción inversa, siguiendo el proceso propuesto por Ramada-Rodilla, Serra-Pujadas y Delclós-Clanchet (2013). En la primera etapa, la escala original en inglés fue traducida por dos traductoras (bilingües español-inglés) con lengua materna española e inscritas en el Colegio de Traductores del Perú, quienes realizaron las traducciones de la versión original al español de forma independiente $\mathrm{y}$, después de revisar las diferencias y similitudes, se acordó una versión síntesis. Ambas traductoras no informaron dificultades en el proceso de traducción debido al lenguaje sencillo del cuestionario. Como segunda etapa de este proceso, la versión síntesis fue retro-traducida por dos traductoras de lengua materna inglesa y que pertenecían a la Asociación de Traductores Profesionales del Perú, quienes no tuvieron acceso a la versión original en inglés de la escala CAMM y realizaron las traducciones inversas de forma independiente.

En la tercera fase de este proceso, las traducciones inversas fueron comparadas con la versión original del inglés del instrumento CAMM por profesores de educación básica regular, nativos de países de habla inglesa, con el objetivo de analizar la equivalencia semántica y no solamente literal entre los ítems de la escala original y las dos versiones traducidas. Se halló que las versiones retro-traducidas y la versión original fueron 
equivalentes conceptual y semánticamente, y se obtuvo un índice de acuerdo de $100 \%$ en todos los casos.

Como siguiente etapa en este proceso de adaptación lingüística, la escala fue revisada por un comité conformado por dos psicólogas y dos profesoras de educación básica regular, bilingües, con experiencia en la aplicación de técnicas de mindfulness en su práctica laboral. Revisaron la adaptación lingüística, la construcción gramatical y el vocabulario de los enunciados de tal manera que estuvieran adecuados a la población a la cual va dirigida la escala CAMM. Los cambios que se efectuaron para incrementar la claridad de los ítems estuvieron referidos principalmente a la redacción y al vocabulario empleado. Luego de realizar tales cambios se concluyó con la versión final de la escala de medida de atención plena para niños y adolescentes.

Posteriormente, se realizó una prueba piloto del instrumento con un grupo de 30 alumnos con características sociodemográficas similares a la muestra total.

Cabe resaltar que se envió previamente una comunicación electrónica para solicitar la autorización a la Dra. Greco, autora de la escala CAMM, para el empleo del instrumento y del procedimiento de adaptación, quien respondió brindando su anuencia por correo electrónico.

\section{Procedimiento}

Los estudiantes que participaron se encontraban matriculados en el año 2018 y asistían a colegios cuyos directores otorgaron la autorización formal a los investigadores para la aplicación de la escala CAMM en sus instituciones, a partir del conocimiento de los propósitos del estudio.

La administración de la escala CAMM se realizó de forma grupal y anónima dentro del es- tablecimiento escolar, durante el horario de clases y en una sesión de aproximadamente 10 a 15 minutos. El protocolo de aplicación iniciaba con la explicitación del objetivo del estudio e implicancias en cuanto a la participación, para obtener así el asentimiento informado, luego se procedía al llenado de la ficha de datos generales y finalmente, al completamiento de la escala CAMM. La recolección de los datos duró cuatro meses, es decir entre junio y septiembre del 2018.

\section{Análisis de datos}

Se siguieron los estándares para la evaluación educativa y psicológica planteados por la American Educational Research Association (AERA), American Psychological Association (APA), y el National Council on Measurement in Education (NCME; 2014).

Con el fin de estudiar la estructura interna de la escala CAMM y determinar el número de factores, se realizó un análisis factorial exploratorio con la primera mitad de la muestra dividida aleatoriamente y luego un análisis factorial confirmatorio con la segunda mitad. Además, para la determinación de la invarianza de la medición de acuerdo con el sexo, se evaluó la invarianza configuracional, invarianza débil e invarianza fuerte (Byrne, 2008) con un estimador robusto (WLSMV; Brown, 2015).

La estimación de la confiabilidad se obtuvo con el coeficiente alfa ordinal y con el análisis correlacional ítem-test, ambos indicadores de la consistencia interna de la medida.

Como otras fuentes de validez en relación con otras variables se analizaron inferencialmente las diferencias según sexo y edad, para ello se emplearon los estadísticos $t$ de Student y ANOVA. 
Resultados

Evidencias de validez vinculadas al contenido de la adaptación

Para establecer las evidencias de validez de contenido de la versión adaptada del CAMM se llevó a cabo el procedimiento de juicio de expertos a través del método de calificación de ítems (Abad, Olea, Ponsoda, \& García, 2011) en el que participaron nueve jueces. Estos fueron seleccionados de acuerdo con su competencia en el ámbito profesional (psicólogos y psiquiatras) y su formación académica y metodológica (magister y doctores) en relación con el constructo. Para determinar la representatividad del constructo se calcularon los coeficientes de $\mathrm{V}$ de Aiken para cada ítem traducido a partir de la calificación otorgada por los jueces, evidenciando así que la adaptación realizada cumple con las características de equivalencia (lingüística y cultural), además de establecer su pertinencia como indicadores representativos del constructo a medir (mindfulness). Los coeficientes $\mathrm{V}$ de Aiken calculados para cada ítem del CAMM ascendieron a .89 (ítem 10), .92 (ítem 2), .94 (ítems 1 y 4), .97 (ítems 3, 5 y 7) y 1.00 (ítems 6, 8 y 9), siendo todos estadísticamente significativos $(p<.05)$. Los 10 reactivos pasaron el criterio de los jueces, dado que obtuvieron índices mayores a .80 (Aiken, 1980, 1985; Escurra-Mayaute, 1988).

\section{Análisis descriptivo de items}

A partir de las respuestas obtenidas se llevó a cabo el análisis descriptivo preliminar de ítems para evidenciar las condiciones previas para los análisis psicométricos (Ferrando \& Anguiano-Carrasco, 2010). Para ello se calcularon la media (M) y la desviación estándar (DE) y para determinar la normalidad en cuanto a sus respuestas se tomó como criterio que los índices de asimetría (As.) y de curtosis $(\mathrm{Cu}$.) se encontraran dentro del rango de +/- 1.5 (Pérez \& Medrano, 2010). Así, los 10 ítems presentaron una distribución normal dado que todos se encontraron dentro del rango criterio establecido, lo cual evidenció la pertinencia de realizar el análisis factorial, tanto exploratorio como confirmatorio.

\section{Tabla 1}

Análisis factorial exploratorio de la escala adaptada CAMM con 10 ítems.

\begin{tabular}{|c|c|c|c|}
\hline Ítem & $\mathbf{M}$ & $\mathbf{D E}$ & Factor 1 \\
\hline Ítem 1 & 2.59 & 1.13 & .70 \\
\hline Ítem 2 & 3.10 & 1.06 & .29 \\
\hline Ítem 3 & 2.55 & 1.17 & .58 \\
\hline Ítem 4 & 2.40 & 1.23 & .69 \\
\hline Ítem 5 & 1.72 & 1.20 & .26 \\
\hline Ítem 6 & 2.33 & 1.24 & .38 \\
\hline Ítem 7 & 2.59 & 1.21 & .78 \\
\hline Ítem 8 & 1.85 & 1.18 & .57 \\
\hline Ítem 9 & 2.44 & 1.22 & .66 \\
\hline Ítem 10 & 2.18 & 1.27 & .34 \\
\hline & & Autovalor & 3.66724 \\
\hline & & Varianza explicada & $54.7 \%$ \\
\hline & & Determinante & .0974 \\
\hline \multicolumn{4}{|c|}{$\mathrm{KMO}=.80 ;$ Prueba de esfericidad de Bartlett $\chi^{2}=2400.1 ; g l=45 ; p<.001$} \\
\hline
\end{tabular}


Evidencias de validez vinculadas a la estructura interna

Análisis factorial exploratorio (AFE). Antes de la realización del AFE se calculó el estadístico de adecuación muestral de Kaiser-Meyer-Olkin (KMO) cuyo valor fue de .85 y se estimó la prueba de esfericidad de Bartlett, que arrojó un indicador significativo. Ambos evidenciaron la adecuación de los datos para proceder con este tipo de técnica (Campo-Arias, Herazo, \& Oviedo, 2012). Para determinar el número de factores a extraer se empleó el método paralelo de Horn (1965) y el método de mínimos cuadrados no ponderados (ULS; Lorenzo-Seva \& Ferrando, 2007) basado en una matriz de correlaciones policóricas (Lloret-Segura, Ferreres-Traver, Hernández-Baeza, \& Tomás-Marco, 2014).

En la Tabla 1 se presentan los resultados del AFE con los 10 ítems de la escala CAMM adaptada. Se identifica que en esta subyace una dimensión, que explica el 54.7\% de la varianza, además se puede apreciar que el ítem 2 (En el colegio, camino de una clase a otra sin darme cuenta de lo que estoy haciendo) y el ítem 5 (Alejo los pensamientos que no me gustan) presentaron cargas factoriales menores a .30. A partir de estos indicadores se consideró pertinente volver a realizar este análisis excluyendo estos dos ítems (Lloret-Segura et al., 2014).

En la Tabla 2 se presentan los resultados del reanálisis, donde los indicadores obtenidos evidencian unidimensionalidad, siendo los valores de las cargas (con respecto al factor extraído) entre .30 (ítem 10) y .80 (ítem 7), es decir mayores a .30 , valor criterio para considerar la pertinencia y relevancia de un ítem con respecto al factor (Lloret-Segura et al., 2014).

Análisis factorial confirmatorio (AFC). Para confirmar la estructura de un solo factor identificado en AFE, se realizó un AFC usando la estimación de máxima verosimilitud en la segunda mitad del conjunto de datos divididos. En la Tabla 3 se presentan los resultados del AFC, para el cual las cargas estimadas con respecto al factor fueron mayores a .30, siendo el rango obtenido entre .32 (ítem 10) y .93 (ítem 7), lo que evidencia la pertinencia y relevancia de los 8 ítems (Pérez-Gil et al., 2000). Estos resultados mostraron un ajus-

Tabla 2

Análisis factorial exploratorio de la escala adaptada CAMM con 8 ítems.

\begin{tabular}{|c|c|c|c|}
\hline Ítem & M & DE & Factor 1 \\
\hline Ítem 1 & 2.59 & 1.13 & .71 \\
\hline Ítem 3 & 2.56 & 1.17 & .56 \\
\hline Ítem 4 & 2.39 & 1.23 & .70 \\
\hline Ítem 6 & 2.34 & 1.24 & .36 \\
\hline Ítem 7 & 2.59 & 1.21 & .79 \\
\hline Ítem 8 & 1.85 & 1.18 & .57 \\
\hline Ítem 9 & 2.44 & 1.22 & .66 \\
\hline Ítem 10 & 2.19 & 1.27 & .30 \\
\hline & & Autovalor & 3.46682 \\
\hline & & Varianza explicada & $74.0 \%$ \\
\hline & & Determinante & .1626 \\
\hline \multicolumn{3}{|c|}{$\mathrm{KMO}=0.84 ;$ Prueba de esfericidad de Bartlett $\chi^{2}=1873.8 ; g l=28 ; p<.001$} & \\
\hline
\end{tabular}


Tabla 3

Cargas factoriales del análisis confirmatorio e índices de ajuste.

\begin{tabular}{|c|c|c|c|c|c|}
\hline \multirow[b]{2}{*}{ Factor } & \multirow[b]{2}{*}{ Indicador } & \multirow[b]{2}{*}{ Estimado } & \multirow[b]{2}{*}{ Error estándar } & \multicolumn{2}{|c|}{ 95\% Intervalo de Confianza } \\
\hline & & & & Inferior & Superior \\
\hline \multirow{8}{*}{ Factor 1} & Ítem 1 & .78 & .02 & .73 & .83 \\
\hline & Ítem 3 & .62 & .03 & .57 & .67 \\
\hline & Ítem 4 & .80 & .03 & .75 & .85 \\
\hline & Ítem 6 & .38 & .03 & .32 & .43 \\
\hline & Ítem 7 & .93 & .03 & .89 & .98 \\
\hline & Ítem 8 & .61 & .03 & .56 & .66 \\
\hline & Ítem 9 & .78 & .02 & .72 & .83 \\
\hline & Ítem 10 & .33 & .03 & .27 & .39 \\
\hline \multirow{2}{*}{ Índices de ajuste } & CFI & TLI & SRMR & RMSEA & RMSEA 95\% IC \\
\hline & .94 & .92 & .04 & .072 & {$[0.0637 ; 0.0800]$} \\
\hline $\begin{array}{l}\text { Valor aceptable } \\
\text { de ajuste }\end{array}$ & $>.90$ & $>.095$ & $<.080$ & $<.080$ & \\
\hline
\end{tabular}

Nota. CFI: ajuste comparativo. TLI: índice Tucker-Lewis. SRMR: raíz del cuadrado medio del residuo. RMSEA: raíz de cuadrados medios del error.

te aceptable (Abad et al., 2011), así el índice de Tucker-Lewis (TLI) fue de .92, el índice de ajuste comparativo (CFI) ascendió a .94, la raíz del cuadrado medio del residuo fue de .036 y la media de la raíz error cuadrado de aproximación (RMSEA) fue de .08 .

En base a los índices de ajuste obtenidos se concluye que estos son adecuados para cada indicador, y las cargas factoriales asociadas brindan evidencia a favor del modelo de un factor.

Con respecto al análisis de la invarianza de la medición de acuerdo con el sexo, se obtuvieron a nivel de la invarianza configuracional $\chi^{2}=$ 199.9 , CFI $=.95$, RMSEA $=0.61$, a nivel de la invarianza débil $\chi^{2}=214.8, \mathrm{CFI}=.94, \Delta \mathrm{CFI}=.003$, $\mathrm{RMSEA}=0.58$ y a nivel de la invarianza fuerte $\chi^{2}=276.8, \mathrm{CFI}=.92, \Delta \mathrm{CFI}=.009$, RMSEA $=$ 0.61 ; en base a estos índices se demuestra dicha invarianza (Cheung \& Rensvold, 2002; Tabla 4).

Estimación de la confiabilidad. La confiabilidad de las puntuaciones derivadas del CAMM adapta-

Tabla 4

Análisis de invarianza factorial según sexo.

\begin{tabular}{lccccc}
\hline \multicolumn{1}{c}{ Modelo } & $\chi^{2}$ & $\boldsymbol{g} \boldsymbol{l}$ & CFI & $\Delta$ CFI & RMSEA \\
\hline Hombres & $67.0^{*}$ & 20 & .941 & & .059 \\
Mujeres & $132.2^{*}$ & 20 & .946 & & .063 \\
1. Configuracional & 199.9 & 40 & .945 & & .061 \\
2. Débil & 214.8 & 47 & .942 & .003 & .058 \\
3. Fuerte & 276.1 & 54 & .924 & .009 & .061 \\
\hline
\end{tabular}

Nota. CFI: ajuste comparativo. TLI: índice Tucker-Lewis. SRMR: raíz del cuadrado medio del residuo. RMSEA: raíz de cuadrados medios del error. 
do se obtuvo mediante el método de consistencia interna, así el valor alfa ordinal estimado fue de .78 para los 8 ítems, el cual puede ser considerado aceptable (Elosua \& Zumbo, 2008). Además, se calcularon los coeficientes alfa ordinales para hombres y para mujeres, estimaciones que ascendieron a .76 y a .80 respectivamente.

Adicionalmente se calculó el coeficiente omega de McDonald para determinar la confiabilidad del factor, y se obtuvo el valor de .81 el cual es considerado aceptable.

Con respecto al análisis de ítems los índices de homogeneidad calculados para cada reactivo superaron el criterio establecido por Kline (1998; $\left.r_{i t}>.20\right)$, dichos índices se encontraron en el rango de .23 (ítem 10) a .63 (ítem 7), evidenciando así la pertinencia de los 8 ítems como indicadores de la escala.

\section{Análisis complementarios}

Se calcularon las puntuaciones en mindfulness para cada participante a partir de la suma ponderada de los 8 ítems que conformaron la escala final adaptada, y se obtuvo la media (M $=16.8)$, la mediana $(\mathrm{Mdn}=17)$ y la desviación estándar ( $\mathrm{DE}=5.52)$. Según el cálculo del estadístico Shapiro-Wilk (W) las puntuaciones en la variable de estudio no presentaban una distribución normal (Razali \& Wah, 2011).

Se evidenciaron diferencias estadísticamente significativas $(U=396694 ; p<.001)$ en los niveles de mindfulness según sexo, pues los varones $(\mathrm{M}=18.0 ; \mathrm{Mdn}=18)$ presentan un mayor grado de mindfulness que las mujeres $(\mathrm{M}=16.2$; $\operatorname{Mdn}=16$ ).

Al comparar el grado de mindfulness según la edad de los participantes (de 10 años a 17 años) se obtuvieron diferencias estadísticamente significativas $\left(\chi^{2}=110 ; g l=7 ; p<.001\right)$ entre las pun- tuaciones promedio. Considerando la mediana (Mdn) en la escala de mindfulness, se evidencia que, a mayor edad, menor capacidad en atención plena.

\section{Discusión}

En función del objetivo general planteado se adaptó lingüísticamente y se analizaron las propiedades psicométricas de la escala Child and Adolescent Mindfulness Measure (CAMM; Greco et al., 2011) en una muestra de niños y adolescentes entre 10 y 17 años, de educación básica regular de Lima Metropolitana. La escala en la versión en español cuenta con propiedades psicométricas de validez y confiabilidad en congruencia con los estudios previos que han presentado versiones adaptadas en varios idiomas y en diferentes contextos (Chiesi et al., 2016; Cunha et al., 2013; de Bruin et al., 2013; Dion et al., 2017; Saggino et al., 2017; Viñas et al., 2015).

La escala fue adaptada lingüísticamente, para lo cual se siguió un proceso riguroso de traducción y adaptación del instrumento original en inglés al español, siguiendo las recomendaciones de la literatura científica (Beaton et al., 1998; Fernández, Pérez, Alderete, Richaud, \& Fernández-Liporace, 2010; Ramada-Rodilla et al., 2013). El método de traducción y retro-traducción ha sido utilizado en otros estudios psicométricos del CAMM para obtener las versiones en otros idiomas y así garantizar la equivalencia semántica y conceptual del instrumento (Chiesi et al., 2016; Cunha et al., 2013; de Bruin et al., 2013; Dion et al., 2017; Saggino et al., 2017; Viñas et al., 2015). En correspondencia con ello, en este trabajo se siguieron las fases propuestas por Ramada-Rodilla et al. (2013) para garantizar la calidad del proceso.

Como parte del proceso de traducción, 
adaptación cultural y validación, (Ramada et al., 2013) la escala fue evaluada por jueces para obtener evidencias en relación con la pertinencia y representatividad de los ítems.

Las evidencias basadas en el contenido a través del criterio de jueces indicaron la pertinencia y relevancia de todos los ítems traducidos; sin embargo, a partir de las respuestas de las participantes obtenidas mediante la aplicación del cuestionario, el análisis factorial evidenció que los ítems 2 y 5 no contaban con indicadores apropiados que permitieran mantenerlos en una escala unidimensional. Sin embargo, estos hallazgos deben ser considerados preliminares y constituyen un punto de partida para estudios posteriores con este instrumento que permitan arribar a conclusiones más definitivas. En el análisis de la estructura interna del CAMM, según los resultados obtenidos a partir del análisis factorial exploratorio y del confirmatorio la escala adaptada para una muestra de estudiantes de Lima Metropolitana revela una estructura unifactorial, corroborando los hallazgos de Greco et al. (2011) y de los estudios psicométricos posteriores realizados en diferentes países (Chiesi et al., 2016; Cunha et al., 2013; de Bruin et al., 2013; Dion et al., 2017; Kuby et al., 2015; Saggino et al., 2017; Viñas et al., 2015). Los resultados obtenidos sugieren que la escala CAMM tiene una estructura unidimensional, es decir, que un solo factor latente parece representar de forma global el nivel de mindfulness disposicional en los sujetos evaluados.

La escala CAMM en su versión adaptada de 8 ítems en español supera ampliamente los criterios para considerar un instrumento como unidimensional (Abad et al., 2011), ya que el factor único extraído explica un $74 \%$ de la varianza (Abad et al., 2011).

En estudios anteriores ya sea en su versión de 10 ítems (Greco et al., 2011; de Bruin et al., 2013; Dion et al., 2017; Kuby et al., 2015; Viñas et al., 2015), de 9 (Cunha et al., 2013) o de 8 ítems (Chiesi et al., 2016; Saggino et al., 2017) se ha evidenciado igualmente la estructura unifactorial de esta escala.

Greco et al. (2011) plantean que, a pesar de haber partido de una conceptualización multidimensional de mindfulness en la construcción del instrumento, esta variable, entre los 10 y 17 años, representa un constructo único asociado con la habilidad de actuar con atención y con la de no juzgar las experiencias del momento presente. Tales habilidades parecen no diferenciarse claramente en este periodo de edad, mientras que, en el caso de los adultos, sí se pueden demostrar cinco facetas claramente distinguibles (Baer et al., 2006). Por otro lado, las otras escalas de medición de atención plena, una para niños (MASS-C) y otra para adolescentes (MASS-A) revelan también una estructura unifactorial (Brown et al., 2011; Lawlor et al., 2014) en concordancia con lo postulado por Greco et al. (2011).

Por otra parte, según el resultado del análisis factorial exploratorio de la escala CAMM adaptada, dos ítems (número 2 y número 5) presentaron cargas factoriales menores al valor mínimo requerido (Nunnaly \& Bernstein, 1994). En primer lugar, el ítem 2 (En el colegio, camino de una clase a otra sin darme cuenta de lo que estoy haciendo) presentó una carga factorial no aceptada según los criterios mínimos establecidos (Nunnaly \& Bernstein, 1994). Estos resultados no concuerdan con los hallados en el estudio de Greco et al. (2011) cuando construyeron la escala, ni con otros posteriores (de Bruin et al., 2013; Cunha et al., 2013; Viñas et al., 2015; Kuby et al., 2015). Sin embargo, tanto Saggino et al. (2017) como Chiesi et al. (2016) informan cargas factoriales por debajo del mínimo aceptable para este reactivo. En ambas investigaciones, estos autores concluyen que el contenido de este ítem no es representativo para la realidad de la escuela italia- 
na, ya que los estudiantes no cambian de salones para sus clases; esta situación es similar a lo que sucede en nuestro contexto, en el que los alumnos en los colegios públicos y en algunos privados de Lima no tienen que trasladarse de un salón a otro para atender a sus cursos.

En segundo lugar, según el análisis factorial exploratorio, en el estudio actual, el reactivo número 5 (Alejo los pensamientos que no me gustan) presentó igualmente una carga factorial por debajo del mínimo requerido (Nunnaly \& Bernstein, 1994) al igual que lo informado por otros investigadores (Chiesi et al., 2016; Cunha et al., 2013; Saggino et al., 2017; Viñas et al., 2015). Por ejemplo, Cunha et al. (2013) encontraron que este enunciado no mostró una performance adecuada en la versión portuguesa y proponen una escala con 9 ítems. Asimismo, Viñas et al. (2015) obtuvieron el mismo resultado al hallar una carga factorial baja para este ítem 5; sin embargo, lo mantuvieron en la versión catalana de 10 ítems.

Siguiendo con el análisis del enunciado 5 de la escala CAMM, tanto Saggino et al. (2017) como Chiesi et al. (2016) hallan que este tiene una carga factorial muy baja y concluyen que la versión de la escala CAMM compuesta por 8 ítems ( $\sin$ los mencionados reactivos números 2 y 5) constituye una herramienta de medición más confiable y apropiada para niños y adolescentes italianos. Del mismo modo que Saggino et al. (2017) y Chiesi et al. (2016) este estudio propone la versión del CAMM adaptada para Lima Metropolitana excluyendo tales reactivos. Los índices del análisis factorial confirmatorio evidencian que la versión de 8 ítems presenta un mejor ajuste de acuerdo con los criterios establecidos para este tipo de análisis que la versión con 10 reactivos (Abad et al., 2011).

Los hallazgos de esta investigación presentan evidencias de validez en relación con la estructura interna de la versión adaptada del CAMM con 8 ítems, que a través de los análisis factoriales exploratorio y confirmatorio ha presentado indicadores de ajuste superiores a los de la versión original de 10 ítems.

La escala CAMM adaptada en la versión en español ha demostrado tener una adecuada consistencia interna, hallándose resultados análogos con los valores obtenidos en el estudio preliminar de Greco et al. (2011) y con los realizados posteriormente (Chiesi et al., 2016; Cunha et al., 2013; de Bruin et al., 2013; Dion et al., 2017; Kuby et al., 2015; Saggino et al., 2017; Viñas et al., 2015). El coeficiente alfa de Cronbach, valor que calcula la consistencia interna de la escala total (Alarcón, 2008) fue determinado tanto para la escala con 10 ítems como para la compuesta por 8 ítems (excluyendo el 2 y 5) en concordancia los estudios realizados con muestras de estudiantes italianos (Chiesi et al., 2016; Saggino et al., 2017).

Al analizar la variable sexo, se encontraron diferencias significativas en los niveles de mindfulness a favor de los hombres, no obstante, los hallazgos en los estudios psicométricos previos del CAMM demuestran cierta divergencia en cuanto a este punto ya que en algunos no se reportaron variaciones entre varones y mujeres (Greco et al., 2011; Kuby et al., 2015; Saggino et al., 2017; Viñas et al., 2015) mientras que en otros sí se evidenciaron (de Bruin et al., 2013; Dion et al., 2017). Se observa entonces que Dion et al. (2017) indicaron diferencias entre adolescentes hombres y mujeres provenientes de escuelas rurales en cuanto a la variable estudiada, sin embargo, no hallaron estas diferencias en la muestra de adolescentes de colegios urbanos. Por otra parte, de Bruin et al. (2014), en el estudio de validación que realizaron en Portugal, obtuvieron igualmente diferencias significativas a favor de los hombres en la muestra de adolescentes; sin embargo, en el caso de la muestra conformada por niños no se registraron tales diferencias. En otras in- 
vestigaciones como la de Cunha y Paiva (2012) quienes estudiaron en una muestra de adolescentes, las variables de mindfulness, ansiedad frente a exámenes y sentimientos de inadecuación frente al fracaso, informaron un mayor nivel del rasgo mindfulness en los hombres. Igualmente, Robinson et al. (2014) utilizaron la escala CAMM para estudiar la relación entre niveles de mindfulness y consumo de alcohol en una muestra de adolescentes e informaron que los varones puntuaron significativamente más alto que las mujeres. Asimismo, Brown et al. (2011) utilizando otros instrumentos de medición como la medida de atención plena MAAS-A hallaron que los adolescentes de sexo masculino obtuvieron puntajes ligeramente más altos en los niveles de mindfulness que en el caso de las mujeres. En este estudio, las mujeres presentaron menores niveles de mindfulness disposicional. Debido a que los hallazgos en la literatura académica no son consistentes, Dion et al. (2017) y Kuby et al. (2015) sugieren realizar más investigaciones que apunten al análisis específico en distintas poblaciones para arribar a evidencias más concluyentes acerca de la capacidad de mindfulness disposicional en cuanto al sexo.

En cuanto al análisis de la variable edad, se encontraron diferencias estadísticamente significativas entre las puntuaciones promedio en el grado de mindfulness, es decir, a mayor edad, menor capacidad en atención plena. Este hallazgo no es consistente con lo que se obtuvo en otros estudios psicométricos que no encontraron diferencias al considerar la edad (Cunha et al., 2013; de Bruin et al., 2013; Greco et al., 2011; Viñas et al., 2015).

Si bien en este estudio no se ha trabajado con niños de 9 años, son interesantes los resultados informados por Lawlor et al. (2014) cuando validaron la Escala de Medida de Atención Plena en una muestra de niños entre 9 y 12 años; dichos autores hallaron que el nivel de mindfulness decrecía con la edad, y sugirieron que los cambios cognitivos y sociales, así como el incremento de las demandas del medio, podrían explicar esta disminución. Adicionalmente, señalan que se requiere de un mayor número de investigaciones en el campo de las neurociencias que puedan explicar el desarrollo de este constructo (Lawlor et al., 2014), teniendo en cuenta que la adolescencia se caracteriza por una etapa de reorganización cerebral (Giedd, 2008). Por otro lado, al encontrarse esta población específica en un proceso de maduración cerebral y desarrollo de sus habilidades cognitivas y socioemocionales, se necesitan de estudios longitudinales para establecer afirmaciones concluyentes en relación con los cambios evolutivos que pueden darse en esta capacidad disposicional.

Se reconoce la existencia de algunas limitaciones. No se obtuvieron evidencias de validez basadas en la relación de mindfulness con otros constructos (convergente y divergente) a diferencia de otros estudios a nivel internacional, dado que las mediciones empleadas en los antecedentes no se encuentran validadas en el contexto peruano para este grupo etario. Cabe considerar además que, si bien la muestra utilizada en este estudio estuvo constituida por 2120 estudiantes, no se realizó un muestreo probabilístico para conformarla, lo cual limita la generalización de los resultados.

A pesar de las limitaciones, este estudio ha sido el primero en analizar las propiedades psicométricas de este instrumento y ha contribuido con el proceso de validación de la escala CAMM en el contexto peruano con una muestra de escolares. Se considera importante contar con una herramienta que mida mindfulness rasgo en niños $\mathrm{y}$ adolescentes debido al valor que ha adquirido este constructo en los últimos años y a su rápida difusión en los ámbitos clínicos y educacionales.

Se sugiere continuar con el análisis de las propiedades psicométricas de la escala CAMM 
en otros contextos y/o grupos etarios, con el objetivo de determinar si los hallazgos de este estudio pueden ser replicados. Será relevante incluir muestras clínicas que presenten sintomatología física, conductual y/o emocional.

\section{Referencias}

Abad, F. J., Olea, J., Ponsoda, V., \& García, C. (2011). Medición en ciencias sociales y de la salud. Madrid: Síntesis.

Aiken, L. R. (1980). Content validity and reliability of single items or questionnaires. Educational and Psychological Measurement, 40(4), 955-959. doi: 10.1177/001316448004000419

Aiken, L. R. (1985). Three coefficients for analyzing the reliability and valid-ity of ratings. Educational and Psychological Measurement, 45, 131-142.

Alarcón, R. (2008). Métodos y Diseños de Investigación del Comportamiento. Lima: Universidad Ricardo Palma.

American Educational Research Association, American Psychological Association, \& National Council on Measurement in Education. (2014). Standards for educational and psychological testing. Washington, DC: American Educational Research Association.

Baer, R. A. (2011). Measuring mindfulness. Contemporary Buddhism, 12(1), 241-261. doi: $10.1080 / 14639947.2011 .564842$

Baer, R. A., Smith, G. T., \& Allen, K. B. (2004). Assessment of mindfulness by self-report: The Kentucky Inventory of Mindfulness Skills. Assessment, 11(3), 191-206. doi: 10.1177/1073191104268029

Baer, R. A., Smith, G. T., Hopkins, J., Krietemeyer, J., \& Toney, L. (2006). Using self-report assessment methods to explore facets of mindfulness. Assessment, 13(1), 27-45. doi: 10.1177/1073191105283504

Baer, R. A., Carmody, J., \& Hunsinger, M. (2012). Weekly change in mindfulness and perceived stress in a mindfulness-based stress reduction program. Journal of Clinical Psychology, 68(7), 755-765. doi: 10.1002/ jclp. 21865

Beaton, D., Bombardier, C., Guillemin, F., \& Bosi, M. (1998). Recommendations for the Cross-Cultural Adaptation of Health Measures. American Academy of Orthopaedic Surgeons. Institute for Work and Health.

Bergomi, C., Tschcher, W., \& Kupper, Z. (2013). The assessment of mindfulness with self-report measures: Existing scales and open issues. Mindfulness, 4(3), 191-201. doi: 10.1007/s12671-012-0110-9

Bickman, L. (2006). Peabody Treatment Progress Battery manual [Electronic version]. Recuperado de http:// peabody.vanderbilt.edu/ptpb

Brown, K. W., \& Ryan, R. M. (2003). The benefits of being present: Mindfulness and its role in psychological well-being. Journal of Personality and Social Psychology, 84(4), 822-848. doi: 10.1037/00223514.84.4.822

Brown, K. W., Ryan, R. M., \& Creswell, J. D. (2007). Addressing fundamental questions about mindfulness. Psychological Inquiry, 18(4), 272-281. doi: 10.1080/10478400701703344

Brown, K. W., West, A. M., Loverich, T. M., \& Biegel, G. M. (2011). Assessing adolescent mindfulness: Validation of an adapted Mindful Attention Awareness Scale in adolescent normative and psychiatric populations. Psychological Assessment, 23(4), 10231033. doi: $10.1037 / \mathrm{a} 0021338$

Brown, T. A. (2015). Confirmatory Factor Analysis for Applied Research (2 $2^{\mathrm{a}}$ ed.). New York, NY: Guilford.

Byrne, B. M. (2008). Testing for multigroup equivalence of a measuring instrument: A walk through the process. Psicothema, 20(4), 872-882. Recuperado de http:// www.psicothema.com

Campo-Arias, A., Herazo, E., \& Oviedo, H. C. (2012). Análisis de factores: fundamentos para la evaluación de instrumentos de medición en salud mental. Revista Colombiana de Psiquiatría, 41(3), 659-671. doi: 10.1016/s0034-7450(14)60036-6

Cunha, M., Galhardo, A., \& Pinto-Gouveia, J. (2013). Child and Adolescent Mindfulness Measure (CAMM): Es- 
tudo da caracteristicas psicométricas de versao portuguesa. Psicología: Reflexao e Critica, 26(3), 459468. doi: 10.1590/S0102-79722013000300005

Cunha, M., \& Paiva, M. J. (2012). Text anxiety in adolescents: The role of self-criticism and acceptance and mindfulness skills. The Spanish Journal of Psychology, 15(2) 533-543. doi: 10.5209/rev_SJOP.2012.v15. n2.38864

Cheung, G. W., \& Rensvold, R. B. (2002). Evaluating goodness-of-fit indexes for testing measurement invariance. Structural Equation Modeling, 9(2), 233255. doi: 10.1207/s15328007sem0902_5

Chiesi, F., Dellagiulia, A., Lionetti, F., Bianchi, G., \& Pri$\mathrm{mi}$, C. (2016). Using item response theory to explore the psycometric properties of the italian version of the Child and Adolescent Mindfulness Measure (CAMM). Mindfulness, 8(2), 351-360. doi: 10.1007/ s12671-016-0604-y

De Bruin, E. I., Zijlstra, B. J. H., \& Bögels, S. M. (2013). The meaning of mindfulness in children and adolescents: Further validation of the Child and Adolescent Mindfulness Measure (CAMM) in two independent samples from the Netherlands. Mindfulness, 5(4), 422-430. doi: 10.1007/s12671-013-0196-8

Dion, J., Paquette, L., Daigneault, I., Godbout, N., \& Hébert, M. (2017). Validation of the French version of the Child and Adolescent Mindfulness Measure (CAMM) among samples of French and Indigenous youth. Mindfulness, 9(2), 645-653. doi: 10.1007/ s12671-017-0807-X

Elosua, P., \& Zumbo, B. (2008). Coeficientes de fiabilidad para escalas de respuesta categórica ordenada. Psicothema, 20(4), 896-901. Recuperado de http://www. psicothema.com

Escurra-Mayaute, L. M. (1988). Cuantificación de la validez de contenido por criterio de jueces. Revista de Psicología, 6(1-2), 103-111. Recuperado de http:// revistas.pucp.edu.pe/index.php/psicologia/article/ view/4555

Fernández, A., Pérez, E., Alderete, A., Richaud, M., \& Fernández-Liporace, M. (2010). ¿Construir o adap- tar tests psicológicos? Diferentes respuestas a una cuestión controvertida. Revista Evaluar, 10(1). doi: 10.35670/1667-4545.v10.n1.459

Ferrando, P. J., \& Anguiano-Carrasco, C. (2010). El análisis factorial como técnica de investigación en psicología. Papeles del Psicólogo, 31(1), 18-33. Recuperado de http://www.papelesdelpsicologo.es

Giedd, J. (2008). The teen brain: Insights from neuroimaging. Journal of Adolescent Health, 42(4), 335-343. doi: 10.1016/j.jadohealth.2008.01.007

Greco, L. A., Baer, R. A., \& Smith, G. T. (2011). Assessing mindfulness in children and adolescents. Development and validation of the Child and Adolescent Mindfulness Measure (CAMM). Psychological Assessment, 23(3), 604-614. doi: 10.1037/a0022819

Greco, L. A., Lambert, W., \& Baer, R. A. (2008). Psychological inflexibility in childhood and adolescence: Development and evaluation of the Avoidance and Fusion Questionnaire for Youth. Psychological Assessment, 20, 93-102. doi: 10.1037/1040-3590.20.2.93

Gresham, F. M., \& Elliot, S. N. (1990). Social Skills Rating System. Circle Pines, MN: American Guidance Service.

Horn, J. (1965). A rationale and test for the number of factors in factor analysis. Psychometrika, 30(2), 179185. doi: 10.1007/BF02289447

Johnson, C., Burke, C., Brinkman, S., \& Wade, T. (2017). Development and validation of a multifactor mindfulness scale in youth: The Comprehensive Inventory of Mindfulness Experience - Adolescents (CHIME-A). Psychological Assessment, 29(3), 264-281. doi: $10.1037 /$ pas 0000342

Kline, P. (1998). The new psychometrics: Science, psychology and measurement. London: Routhledge.

Kuby, A. K., McLean, N., \& Allen, K. (2015). Validation of the Child and Adolescent Mindfulness Measure (CAMM) with non-clinical adolescents. Mindfulness, 6(6), 1448-1455. doi: 10.1007/s12671-0150418-3

Lawlor, M. S., Schonert-Reichl, K. A., Gadermann, A. M., \& Zumbo, B. D. (2014). A validation study of 
the Mindful Attention Awareness Scale adapted for children. Mindfulness, 5(6), 730-741. doi: 10.1007/ s12671-013-0228-4

León del Barco, B., Martin-López, E., García-Martin, A., \& Felipe, E. (2008). Estudio preliminar de la escala de atención plena "Mindfulness" en el ámbito escolar. Revista de Psicología, 1, 371-380. Recuperado de http://revistas.pucp.edu.pe/index.php/psicologia/ index

Lloret-Segura, S., Ferreres-Traver, A., Hernández-Baeza, A., \& Tomás-Marco, I. (2014). El análisis factorial exploratorio de los ítems: Una guía práctica, revisada y actualizada. Anales de Psicología, 30(3), 11511169. Recuperado de https://revistas.um.es/analesps/ index

Lorenzo-Seva, U., \& Ferrando, P. (2007). FACTOR: A computer program to fit the exploratory factor analysis model. Behavior Research Methods, 38(1), 8891. doi: 10.3758/bf03192753

Montero, I., \& León, O. G. (2002). Clasificación y descripción de las metodologías de investigación. Psicología Revista Internacional de Psicología Clínica y de la Salud, 2(3), 503-508. Recuperado de https://www. elsevier.es/en-revista-international-journal-of-clinical-and-health-psychology-355

Nunnally, J. C., \& Bernstein, I. H. (1994). Psychometric Theory ( $3^{\mathrm{a}}$ ed.). New York: Mc Graw-Hill, Inc.

Patrick, D. L., Edwards, T. C., \& Topolski, T. D. (2002). Adolescent quality of life, Part II: Initial validation of a new instrument. Journal of Adolescence, 25, 287-300. doi: 10.1006/jado.2002.0471

Pérez, E., \& Medrano, L. (2010). Análisis factorial exploratorio: Bases conceptuales y metodológicas. Revista Argentina de Ciencias del Comportamiento, 2(1), 58-66. Recuperado de https://revistas.unc.edu.ar/index.php/racc

Pérez-Gil, J., Chacón, S., \& Moreno, R. (2000). Validez de constructo: El uso del análisis factorial exploratorio confirmatorio para obtener evidencias de validez. Psicothema, 12(2), 442-446. Recuperado de http:// psicothema.com
Posner, M., Rothbart, M., \& Tang, Y. (2015). Enhancing attention through training. Current Opinion in Behavioral Sciences, 4, 1-5. doi: 10.1016/j.cobeha.2014.12.008

Ramada-Rodilla, J. M., Serra-Pujadas, C., \& Delclós-Clanchet, G. (2013). Adaptación cultural y validación de cuestionarios de salud: Revisión y recomendaciones metodológicas. Salud Pública México, 55(1), 57-66. doi: 10.1590/s0036-36342013000100009

Razali, N., \& Wah, Y. (2011). Power comparison of Shapiro-Wilk, Kolmogorov-Smirnov, Lilliefors and Anderson Darling tests. Journal of Statistical Modeling and Analytics, 2(1), 21-33.

Robinson, J. M., Ladd, B. O., \& Anderson, K. G. (2014). When you see it, let it be: Urgency, mindfulness and adolescent substance use. Addictive Behaviors, 39(6), 1038-1041. doi: 10.1016/j.addbeh.2014.02.011

Saggino, A., Bartoccini, A., Sergi, M. R., Romanelli, R., Maccchia, A., \& Tommasi, M. (2017). Assessing mindfulness on samples of Italian children and adolescents: The validation of the Italian version of the Child and Adolescent Mindfulnes Measure. Mindfulness, 8(5), 1364-1372. doi: 10.1007/s12671-0170712-3

Sauer, S., Walach, H., Schmidt, S. Hinterberger, T., Lynch, S., Büssing, A., \& Kohls, N. (2012). Assessment of mindfulness: Review on state of the art. Mindfulness, 4(1), 3-17. doi: 10.1007/s12671-012-0122-5

Siegel, D. (2010). Cerebro y mindfulness. España: Paidós.

Simón, V. (2007). Mindfulness y neurobiología. Revista de Psicoterapia, 17(66), 5-30. Recuperado de http://ojs. revistadepsicoterapia.com/index.php/rdp/index

Simón, V. (2010). Mindfulness y psicología: Presente y futuro. Información Psicológica, 100, 162-170. Recuperado de http://www.informaciopsicologica.info/ index.php

Viñas, F., Malo, S., González, M., Navaro, D., \& Casas, F. (2015). Assessing mindfulness on a sample of Catalan-speaking Spanish adolescents: Validation of the Catalan version of the Child and Adolescent Mindfulness Measure. Spanish Journal of Psychology, 
18(46), 1-8. doi: 10.1017/sjp.2015.48

Walker, L. S., \& Garber, J. (2001). Manual for the Children's Somatization Inventory. Nashville, TN: Vanderbilt University, Department of Pediatrics.

Wegner, D. M., \& Zanakos, S. (1994). Chronic thought suppression. Journal of Personality, 62, 615-640. doi: 10.1111/j.1467-6494.1994.tb00311.x 